\title{
ON THE DYNAMIC AND QUASI-STATIC SHEAR STRENGTH OF SLM ALSI10MG
}

\author{
Ben Amir ${ }^{* 1}$, Eytan Kochavi ${ }^{1}$, Shimon Gruntman ${ }^{1}$, Yuval Gale $^{3}$, Shmuel Samuha ${ }^{2}$, and \\ Oren Sadot ${ }^{1}$ \\ ${ }^{1}$ Department of Mechanical Engineering, Ben-Gurion University of the Negev, Beer-Sheva 84105, Israel \\ ${ }^{2}$ Department of Materials Engineering, NRCN, P.O. Box 9001, Beer-Sheva 84190, Israel \\ ${ }^{3}$ Additive Manufacturing Center of Excellence, Technologies Division, Israeli
}

\begin{abstract}
Additive manufacturing by selective laser melting (AM-SLM) is an advanced manufacturing approach in which a structure is fabricated by successive thin powder layers melted by a focused laser beam. The aerospace and automotive sectors are especially interested in the AMSLM technology that enables quick production of complex and customized structures. AlSi10Mg alloy has been found to be applicable to AM-SLM mainly because good cast-ability, strong weldability and low shrinkage during solidification. While many studies on the quasi-static mechanical properties and the structure of SLM AlSi10Mg were published, there is limited published research focused on the dynamic properties of SLM AlSi10Mg under high rate strains. In addition to that, the shear strength of SLM aluminium alloys is rarely investigated. This study presents an investigation of the AM-SLM AlSi10Mg static and dynamic shear strength and its dependency on build direction. Experiments included quasi-static shear experiments performed according to the protocol of ASTM B565, and dynamic shear tests performed using a split Hopkinson pressure bar (SHPB), coupled to innovative punch assembly that generates pure dynamic shear loads on the sample. The design of this sample holder has been validated numerically and an experimentally. The quasi-static experiments revealed that the static shear strength is independent of build direction. In contrast, the dynamic tests demonstrated that the dynamic shear strength of vertically built samples is higher by almost $11 \%$ than the shear strength of samples built horizontally. This last phenomenon explained with a suggested mechanism based onelectron microscope fractography.
\end{abstract}

\section{Introduction}

Selective laser melting (SLM) of a powder bed is the most utilized manufacturing technique of additive manufacturing (AM) of metal alloys, also known as 3D printing. The implementation of SLM AlSi10Mg in the aerospace and automotive industries is widespread due to the combination of low density and relatively high strength. The excellent characteristics during casting and welding makes the AlSi10Mg alloy suitable for the SLM process [1]. The SLM process affects the microstructure of the AlSi10Mg leaving microscopic laser metal tracks. The tracks are characterized by a half cylindrical shaped melting zone, similar to larger tracks left by welding aluminum alloy. the melting zone or melt pools (MPs) has a unique visible microstructure with an eutectic Si network at the boundaries and $\alpha-\mathrm{Al}$ at the center [2]. The MPs create a typical fish scale morphology that in turn, enhances the mechanical properties of the product.

Numerous studies which investigated the alloy's quasi-static mechanical properties established that the strength and hardness of the SLM AlSi10Mg alloy is comparable or even higher than those obtained with convential cast alloy[3]. Moreover, under static loads, SLM AlSi10Mg product is independent on build direction and strain rate[4].

Recently, the scientific community became interested in the dynamic behavior of SLM AlSi10Mg. Using a split Hopkinson pressure bar (SHPB) apparatus, it was established that the alloy's dynamic properties are sensitive to fabrication orientation. Specifically, at strain rates in the range from $7 \times 10^{2}$ to $7 \times 10^{3} \mathrm{~s}^{-1}$, vertically built samples had $10 \%$ higher yield stress than samples manufactured horizontally and the alloy's peak stress is also slightly strain rate sensitive [2][5].

\footnotetext{
* Corresponding author: benu@ post.bgu.ac.il
} 
Despite the comprehensive studies on the SLM AlSi10Mg under compression, the shear strength of this alloy received very little attention. Denti [6] investigated the SLM A357.0 shear behavior in respect to the building orientation, and found out that as the orientation of the specimens leans towards vertical orientation, the shear strength increases.

Quasi-static shear testing procedures are well defined by multiple standards; On the other hand, dynamic shear testing was performed by a wide range of methods by different investigators, such as Dorogoy et al. [7], Karp et al. [8] and Gilat et al. [9], all of which used a different type of shear sample or designed an adapter in conjunction with a conventional SHPB system. The above samples or adapters could not apply failure under pure shear. Their experiments had to be assisted by numerical simulations to determine the triaxiality [7] [8] obtained in the experiments [9].

The herein presented study characterizes pure-shear strength of SLM AlSi10Mg under a wide range of loading rates, and presents the shear strength dependency on the build orientation. The quasi-static shear strength is determined according to ASTM B565-04 [10] standard and the dynamic shear has been characterized using a specially designed punch apparatus that was validated numerically and experimentally.

\section{Methods}

\subsection{Samples preparation}

The AlSi10Mg samples were manufactured with an SLM M280 model machine using the optimal parameters recommended by the company [11]. In order to examine the effect of build orientation on shear strength, cylinder shaped bulks, $600 \mathrm{~mm}$ in height and $10 \mathrm{~mm}$ in diameter, were fabricated in two orientations: horizontally (XY) and vertically (Z). From these bulks, samples were machined into two different forms in order to suits the necessary experimental system. For the quasi-static experiment, the samples were machined into $20 \mathrm{~mm}$ in height cylinder with diameter of $8 \mathrm{~mm}$. For the dynamic experiments, the sample were machined into discs, $8.5 \pm 0.1 \mathrm{~mm}$ in diameter and $2 \pm 0.1 \mathrm{~mm}$ thick. The fabrication parameters at the circumference of the layered bulk were different from those at the samples core. Hence, experiments on the samples machined out of the core region, is able to characterize the mechanical properties of the main core of the product without shell effects.

The density of the XY and $\mathrm{Z}$ machined specimens was measured using Archimedes method. The relative density of all the samples was $99.1 \% \pm 0.1 \%$ of the density of fully dense AlSi10Mg alloy, with negligible variance between orientations.

\subsection{Shear experiments}

Shear tests were performed in three rate regimes, quasi static, low rate and dynamic. For each orientation and rate, at least three tests were performed at room temperature. A Lloyd LD50 machines was used for the quasi static and low-range testing. The quasi static experiment were performed according to the ASTM B565-04 [10] standard which requires that "the crosshead speed rate does not exceed $19.1 \mathrm{~mm} / \mathrm{min}$, and the loading rate is maintained under $689 \mathrm{MPa} / \mathrm{min}$ ". Specifically, the normalized displacement rate $(\dot{u})$, which is the displacement velocity divided by the specimen thickness, was $1.6 \times 10^{-2} \mathrm{~s}^{-1}$. The medium-range experiment configuration, was similar to the quasi static experiment however, the $\dot{u}$ was $5 \mathrm{~s}^{-1}$. The dynamic shear strength $\left(\dot{u}=2.3 \times 10^{3} \mathrm{~s}^{-1}\right)$ was determined with a novel design of punch and sample-holder system, presented in Figure 1. This system consists of a cylindrical punch (in green) concentrically placed with the incident bar (in white) and the disk specimen (in yellow). The specimen is fixed between two threaded cylinders supported by an outer ring (in dash blue). The dynamic punch interfaces with the SHPB by placing the punch between the two long bars, details on the SHPB system can be found in our earlier studies [2][5].

The assumptions used to interpret the specimen's shear stress from the strain at the bars is based on the assumptions of pure shear failure, one wave analysis and force equilibrium between the bar's edges. These assumptions were verified numerically and experimentally as detailed in the following chapter.

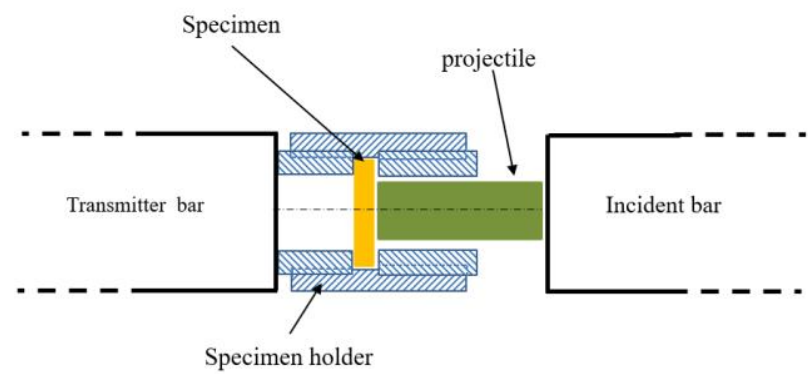

Figure 1. Schematic of the dynamic shear punch assembly coupled to SHPB experimental system 


\section{Validation of the dynamic shear punch}

To validate the above-mentioned assumptions, it was necessary to use a well-known material with established mechanical properties and a widely used material model. For the purpose of the experimental and numerical validation of the current suggested testing method, samples were machined from 6061-T6 aluminum alloy. Once the testing method is validated numerically and experimentally with the above well-known alloy, Formulas (1)(3) will be used in order to analytically calculate the specimen shear stress $\left(\tau_{\text {shear }}\right)$, the normalized displacement $\left(u_{s(t)}\right)$, and normalized displacement rate $\left(\dot{u}_{s(t)}\right)$. This analytical interpretation will enable the direct calculation of $\tau_{\text {shear }}, u_{s_{(t)}}$ and $\dot{u}_{s(t)}$ from the measured strains at the incidence $\left(\varepsilon_{R}\right)$ and the transmitted ( $\left.\varepsilon_{T}\right)$ bars, without having to use numerical computations for each test. The constants used is $A_{B}, E_{S}$ and $C_{B}$ which are the crosssectional area, Young's modulus and elastic wave velocity of the bar respectively. The specimen thickness and sheared area depict by $w$ and $A_{\text {shear }}$ respectively.

$$
\begin{gathered}
\tau_{\text {shear }}=\frac{A_{B} E_{s} \varepsilon_{T}(t)}{A_{\text {shear }}} \\
\dot{u}_{s(t)}=\frac{-2 C_{B} \varepsilon_{R}(t)}{w} \\
u_{s(t)}=-2 \frac{C_{B}}{w} \int_{0}^{t} \varepsilon_{R}(t) d t
\end{gathered}
$$

In order to use those formulas two assumptions are made: the first is force equilibrium between the bars edges and the second is pure shear failure in the samples deformed area. An axisymmetric numerical analysis was implemented in LS-DYNA software using the Johnson-Cook material model. The material model used for the bars, projectile and sample holder are all considered to be linear elastic with mechanical properties of steel. The computational initial conditions were as in the specific test: actual striker velocity $9.1 \mathrm{~m} / \mathrm{s}$ and a tested A16061T6 sample $2.1 \mathrm{~mm}$ thick.

Figure 2 (a) depicts the strains at the bars as a function of time, a dashed line presents the simulated strains and a continuous line for the experimental strains. Despite the reverberations in the simulated signal, there is a satisfied agreement between the lines. The excellent fit suggest that the material parameters used in the model were adequate. From the simulation it can be concluded that the deformed area is limited to the intentional gap between the projectile and the sample holder, defining the sheared zone, in which the triaxiality parameter is lower than 0.08 . Thus, the pure shear mode assumption is verified. Figure 2 (b) shows in a blue line the strain generated by the initial impact in the incident bar $\left(\varepsilon_{\mathrm{I}}\right)$. The orange line depicts the transmitted strain $\left(\varepsilon_{T}\right)$ added to the reflected strain $\left(\varepsilon_{R}\right)$ after interaction with the specimen. The agreement between the lines indicate that $\varepsilon_{I}=\varepsilon_{T}+\varepsilon_{R}$ and validate the assumption of force equilibrium according to the momentum conservation law.

From experiments the maximal shear stress of $209 \pm 2 \mathrm{MPa}$ is directly calculated, and the numerical maximal shear result was $207 \mathrm{MPa}$. The shear stress obtain from the experiments and simulation are in satisfied agreement. Furthermore, those results are in excellent agreement with the shear strength known from literature for al $6061 \mathrm{~T} 6$ - $207 \mathrm{MPa}$ [12]. 

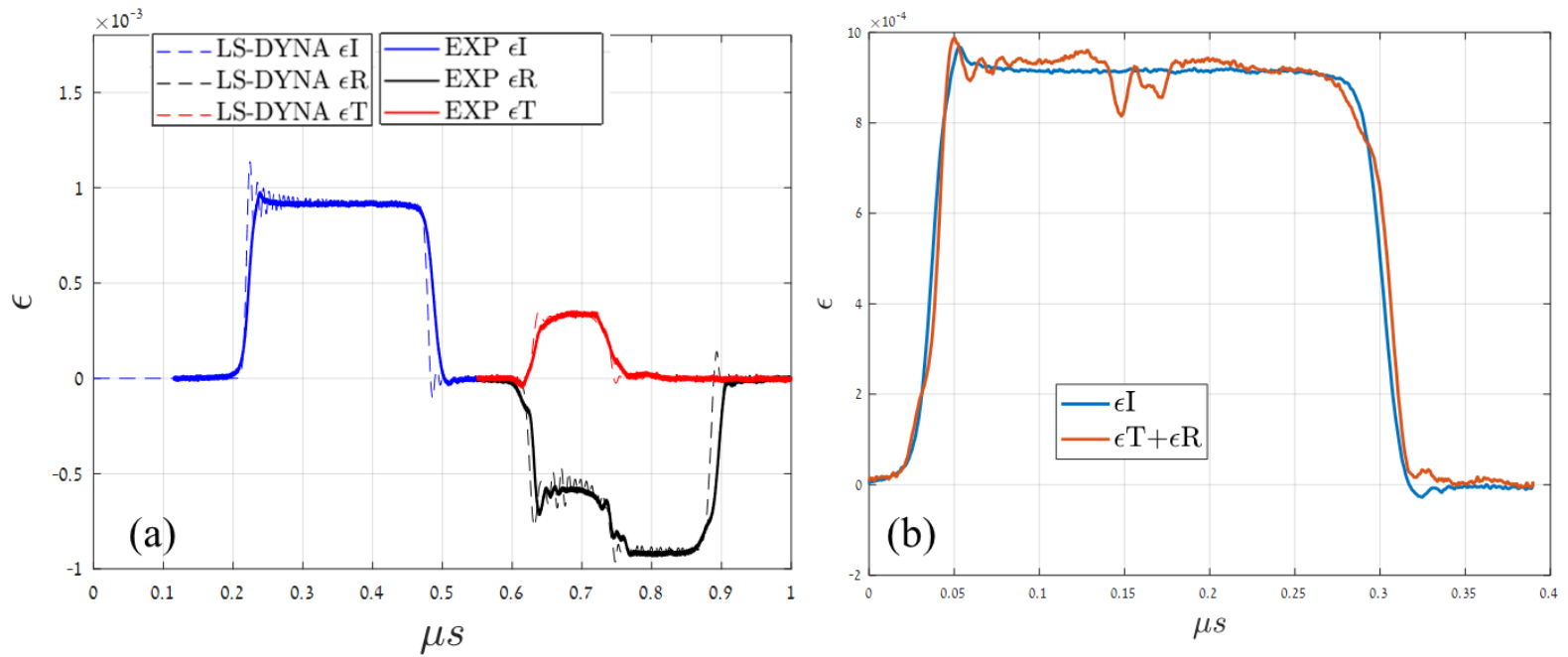

Figure 2. (a)- Comparison between the simulated strains (dashed) and the measured strains (solid), (b)- force equilibrium validation $\varepsilon_{T}+\varepsilon_{R}$ in orange and $\varepsilon_{I}$ in blue

\section{Results and Discussion}

The results shown in Figure 3 (a) and (b) are the mean value of the experimental results, displayed by the shear stress as function of the normalized displacement. The presented results are up to the point of maximum shear stress obtained, which is the alloy's maximal shear strength. The blue and orange lines are for the $\mathrm{Z}$ and XY oriented samples respectively. The dashed line in Figure 3 (a) are the results for the experiments taken strictly according to the ASTM standard at $\dot{u}=1.6 \times 10^{-2} \mathrm{~s}^{-1}$, and the solid line in Figure 3 (a) is for the medium velocity experiments at $\dot{u}=5 \mathrm{~s}^{-1}$.

The shear strength results for the XY oriented samples is $219 \pm 4 \mathrm{MPa}$ for the quasi static loading, and 221 \pm $5 \mathrm{MPa}$ for the medium range loading. The $\mathrm{Z}$ oriented samples shear strength results are $225 \pm 6$ and $227 \pm 7$ for the quasi static and medium range loading respectively. The normalized displacement for all the samples was $0.21 \pm 0.07$. Those results indicate that the SLM AlSi10Mg's shear strength is not influenced by load rate. Moreover, the samples orientation has on influence on the shear strength at quasi static to medium loading rates. The static tests results prove the maximal shear strength and the shear failure strain are within the standard deviation of all tests. At similar loading rates, Rosenthal et al. [4] reported strain rate sensitivity when applying tension loading. This phenomenon was not observed when the SLM AlSi10Mg subjected to shear loading, possibly due to different tensor of stresses.
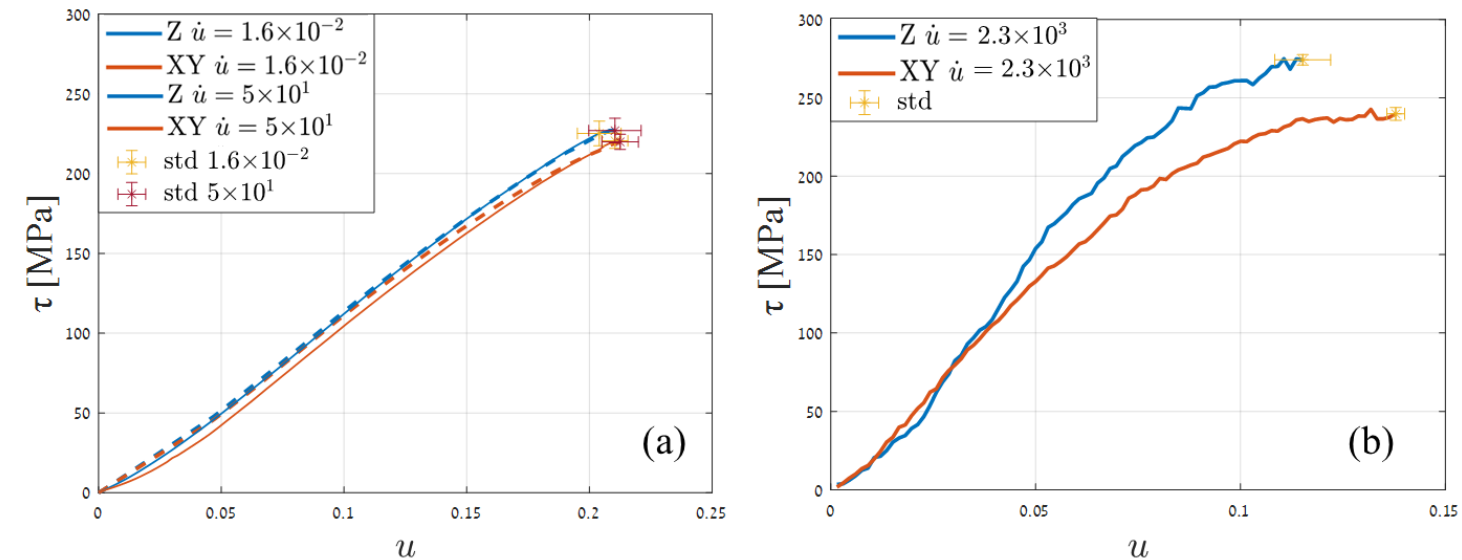

Figure 3. Results of the shear strength vs. normalized displacement for the XY- and Z-(a) under quasi-static and mediumrange loading, (b) under dynamic loading 
Figure 3 (b) shows the dynamic results obtained from the dynamic tests. It can be observed that at high loading rates the shear strength depends on the samples build orientation. Also, for both orientations, the shear strength under dynamic loading is higher than in quasi static and medium-range loading. $Z$ oriented samples are found to be $274 \pm 4 \mathrm{MPa}$ and for the $\mathrm{XY}$ oriented samples is $240 \pm 4 \mathrm{MPa}$. Thus, the dynamic increase of shear strength is approximately $12 \%$ and $21 \%$ for $\mathrm{XY}$ and $\mathrm{Z}$ samples respectively, and is statistically significant using the $\chi 2$ statistical test. The dependency of mechanical properties at high loading rates on the anisotropy of the SLM AlSi10Mg was also reported in our previous work [2]. It was well established that the difference between orientations is associate with the grain morphology of the MPs which in turn, promote the Orowan's affect. In order to characterize the cracks path and failure mode, scanning electron microscopy (SEM) was used. Figure 4 (a) to (c) show the SEM images and a schematic sketch of the crack path for the $\mathrm{Z}$ oriented samples and Figure 4 (d) to (g) for the XY oriented samples. The fractography features presented in the $\mathrm{Z}$ samples Figure 4 (a) and (b) is of brittle fracture with rough edges and quasi-cleavage on the shear surface. Moreover, the quasi-cleavage step pattern is in the size and shape of the MPBs (Figure 4 (a)). In the XY samples, Figure 4 (d) and (e), the sheared dimples are slightly tilted toward the shear direction with fine-sized dimples indicting ductile-brittle fracture. Thus, although both orientations show subtle difference in the fractography features both indicating a pure shear features mode. Figure 4 (c)and Figure 4 (f) is a schematic summarizing of the crack path for $\mathrm{Z}$ and $\mathrm{XY}$ samples respectively. In both samples the crack nucleation is by cleavages in the ductile Si phases. However, at the $\mathrm{Z}$ samples the cracks is propagated between the tracks of the MPs (track-track) and there are secondary cracks. It can be assumed that high amounts of energy deteriorate by the need to deformed the cracks deflection at the $\mathrm{Z}$ samples. At the XY samples the deflection is at smaller angle because the crack nucleation is between the layer interface (layer-layer) enabled more slipping interfaces.
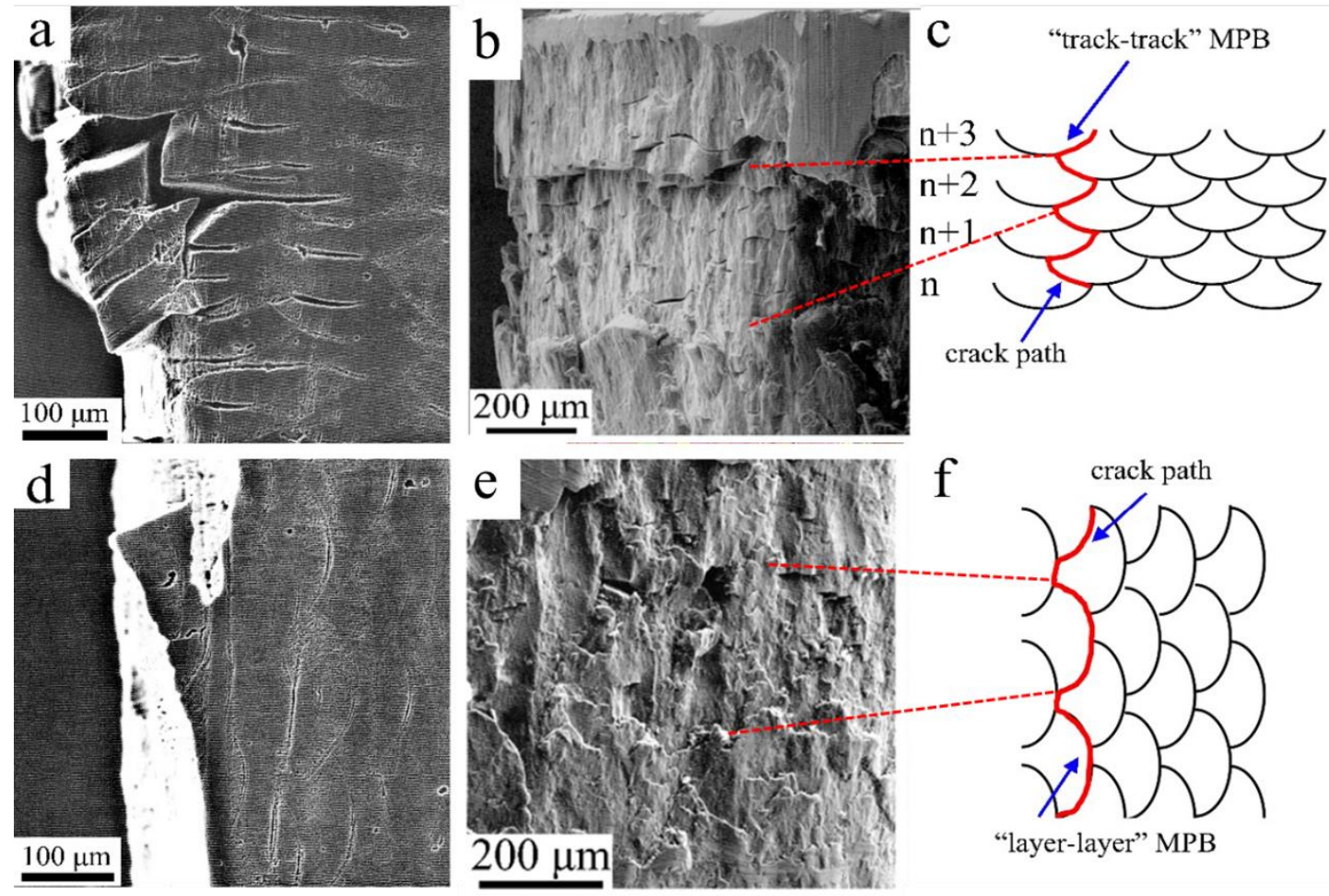

$200 \mu \mathrm{m}$

"layer-layer" MPB

Figure 4. (a), (b) and (d), (e)-SEM images of the fracture surface closest to the shear zone. (c)and (f) scheme of the crack path. Z samples -(a)-(c), XY samples -(d)-(f),

\section{Conclusions}

This study presents the shear behavior of SLM AlSi10Mg under variable loading rate. In order to characterize the dynamic shear, an innovative punch and sample holder device was introduced. the punch was verified to generate pure shear failure and that can be used in a standard SHPB system. The results 
show that in the range of $\dot{u}=1.6 \times 10^{-2}$ to $5 \mathrm{~s}^{-1}$ the AlSi10Mg alloy has isotropic shear behavior and no rate dependency. However, in high rates, shear strength of $Z$ samples increases by $21 \%$ and by $12 \%$ for the XY samples. Thus, under dynamic loading SLM AlSi10Mg is layer-orientation dependent. The deformation mechanism changes according to samples orientations, failure mode of track- track is found in the $\mathrm{Z}$ samples and a crack path of layer- layer at the XY samples. As consequence of this study results, when designing SLM AlSi10Mg parts which undergo shear stress, the orientation and loading rate must be taken into consideration.

\section{References}

1. T. DebRoy, H.L. Wei, J.S. Zuback, T. Mukherjee, J.W. Elmer, J.O. Milewski, A.M. Beese, A. WilsonHeid, A. De, W. Zhang, "Additive manufacturing of metallic components - Process, structure and properties," Prog. Mater. Sci., vol. 92, pp. 112-224, 2018, doi: 10.1016/j.pmatsci.2017.10.001.

2. B. Amir, S. Samuha, and O. Sadot, "Influence of Selective Laser Melting Machine Source on the Dynamic Properties of AlSi10Mg Alloy,” Materials (Basel)., vol. 12, no. 7, p. 1143, 2019, doi: 10.3390/ma12071143.

3. E. Brandl, U. Heckenberger, V. Holzinger, and D. Buchbinder, "Additive manufactured AlSi10Mg samples using Selective Laser Melting (SLM): Microstructure, high cycle fatigue, and fracture behavior," Mater. Des., vol. 34, pp. 159-169, 2012, doi: 10.1016/j.matdes.2011.07.067.

4. I. Rosenthal, A. Stern, and N. Frage, "Strain rate sensitivity and fracture mechanism of AlSi10Mg parts produced by Selective Laser Melting,” Mater. Sci. Eng. A, vol. 682, no. June 2016, pp. 509-517, 2017, doi: 10.1016/j.msea.2016.11.070.

5. B. Nurel, M. Nahmany, N. Frage, A. Stern, and O. Sadot, "Split Hopkinson pressure bar tests for investigating dynamic properties of additively manufactured by selective laser melted AlSi10Mg alloy," pp. 1-28.

6. L. Denti, "Additive manufactured A357.0 samples using the laser powder bed fusion technique: Shear and tensile performance," Metals (Basel)., vol. 8, no. 9, 2018, doi: 10.3390/met8090670.

7. A. Dorogoy, D. Rittel, and A. Godinger, "Modification of the Shear-Compression Specimen for Large Strain Testing," Exp. Mech., vol. 55, no. 9, pp. 1627-1639, 2015, doi: 10.1007/s11340-015-0057-6.

8. B. Karp, A. Dorogoy, and D. Rittel, “A Shear Compression Disk Specimen with Controlled Stress Triaxiality Under Dynamic Loading,” Exp. Mech., vol. 53, no. 2, pp. 243-253, 2013, doi: 10.1007/s11340-012-9630-4.

9. A. Gilat and J. D. Seidt, "Dynamic punch testing of 2024-T351 aluminum," in AIP Conference Proceedings, May 2009, vol. 1195, no. 5, pp. 711-714, doi: 10.1063/1.3295239.

10. ASTM, "B565-04," 2015.

11. SLM Solutions, "Material Data Sheet Al-Alloy AISi10Mg," SLM Solutions Group AG, 2019. https://www.slm-solutions.com/fileadmin/user_upload/MDS_Al-lloy_AlSi10Mg_0619.pdf.

12. G. Totten and D. Scott Mackenzie, Eds., Handbook of Aluminum. CRC Press, 2003. 